\title{
Frontières
}

\section{Apocalypses et fins du monde}

\section{Patrick Bergeron et Joseph Josy Lévy}

Volume 25, numéro 2, 2013

Apocalypses et imaginaires de la fin

URI : https://id.erudit.org/iderudit/1024935ar

DOI : https://doi.org/10.7202/1024935ar

Aller au sommaire du numéro

Éditeur(s)

Université du Québec à Montréal

ISSN

1916-0976 (numérique)

Découvrir la revue

Citer ce document

Bergeron, P. \& Lévy, J. J. (2013). Apocalypses et fins du monde. Frontières, 25(2),

5-10. https://doi.org/10.7202/1024935ar

Ce document est protégé par la loi sur le droit d'auteur. L'utilisation des services d'Érudit (y compris la reproduction) est assujettie à sa politique d'utilisation que vous pouvez consulter en ligne.

https://apropos.erudit.org/fr/usagers/politique-dutilisation/
Cet article est diffusé et préservé par Érudit.

Érudit est un consortium interuniversitaire sans but lucratif composé de l’Université de Montréal, l'Université Laval et l'Université du Québec à Montréal. Il a pour mission la promotion et la valorisation de la recherche. https://www.erudit.org/fr/ 


\title{
APOCALYPSES ET FINS DU MONDE
}

\author{
Patrick Bergeron, Ph. D. \\ Professeur agrégé, Département d'études françaises, \\ Université du Nouveau-Brunswick \\ Joseph Josy Lévy, Ph. D. \\ Professeur, Département de sexologie, \\ Université du Québec à Montréal
}

L'Occident christianisé évolue entre deux fins du monde temporalisées: I'une, le Déluge, est située dans le passé et a le patriarche Noé pour protagoniste; l'autre, le Jugement dernier, appartient à l'avenir et doit servir à départager les damnés et les justes. Lorsqu'il s'agit d'évoquer les derniers jours de l'humanité, la Bible fournit aussi les notions d'apocalypse, d'Armageddon, de dies irae, auxquelles les exégètes préfèrent souvent celle, plus spécifique, d'eschatologie. Les origines religieuses de la fin du monde ne sont toutefois pas exclusives au christianisme, puisqu'on les retrouve, sous d'autres formes, dans le judaïsme, l'islam, de même que dans des cultes plus anciens, tel le zoroastrisme. Au fil des civilisations, les hommes ont imaginé plusieurs scénarios de catastrophes. Bernard Sergent en énumère quelques-uns entre I'âge des Sumériens et les Chroniques gargantuines de 1532 :
inondation colossale ou déluge, [...] selon des modalités variées (eaux célestes ou eaux d'en bas, serpent qui fait déborder la mer, etc.); incendie universel; séisme; basculement de la terre; chute d'un astre ; chute de cendres; chute de montagne embrasée; lutte entre les dieux, ou entre les dieux et leurs adversaires; vent, pluie, grêle, pluie de sable; bêtes carnassières (jaguars, loups); fléchage de toute la population; oiseaux dangereux; invasions de sauterelles; révoltes des objets; courge dévorante; métamorphose des hommes en animaux ${ }^{1}$.

Ces fléaux font penser aux visions présentées dans le Livre de Daniel et L'Apocalypse, deux références fondamentales pour l'imaginaire judéochrétien. Pourtant, les représentations de fin du monde se sont sécularisées, s'imprégnant d'un caractère irréversible et pessimiste absent du modèle biblique. Très présente dans la culture populaire contemporaine, l'apocalypse est devenue «un mot fourre-tout que l'on emploie pour décrire un événement ou un processus particulièrement destructeur ${ }^{2} \gg$. Les horreurs des cent dernières années se sont révélées particulièrement propices au langage apocalyptique : guerres, génocides, désastres écologiques, destruction de la biosphère, épidémies du sida, du SARS... La science-fiction a fait ses choux gras de périls réels ou imaginaires: holocaustes nucléaires, conflits biochimiques, invasions 
extraterrestres... Selon une anecdote répandue (mais sujette à caution), même Einstein aurait prédit l'extinction de l'humanité advenant la disparition des abeilles. Bref, depuis l'aube des temps, les hommes n'ont cessé d'imaginer la fin du monde et d'en réactualiser la menace, comme ce fut le cas en 2012.

Ce numéro de Frontières émane d'un colloque organisé lors du $80^{\circ}$ congrès annuel de l'Acfas, en mai 2012. Nous nous étions inspirés de l'aura apocalyptique conférée à l'année 2012 - et plus particulièrement à la date «fatidique » du 21 décembre - pour proposer une série de réflexions sur la fin du monde et les scénarios de mort universelle qu'elle suscite. Depuis la grande peur de I'an deux mil et, plus encore, les attentats du 11 septembre 2001, la civilisation du «village global» est entrée dans une phase où une multitude d'enjeux économiques, écologiques, géopolitiques et sanitaires sont pensés à partir d'une terminologie ou d'une imagerie apocalyptiques. Un tel consensus entre les discours est un phénomène peu fréquent. Il nous a semblé pertinent de nous interroger à son propos et de chercher un éclairage du côté des sciences religieuses, sociales, médicales et humaines.

Le premier article du numéro situe la question de l'apocalypse dans la pensée islamique. Jean-René Milhot et Frédéric Castel analysent dans un premier temps les textes coraniques et d'autres sources connexes pour y dégager la place du récit apocalyptique. Celui-ci reprend certains des éléments présents dans la théologie chrétienne. On y retrouve les références au jugement dernier, à I'Antéchrist, à Gog et Magog, de même qu'à la Bête. Le Jour du jugement sera ainsi précédé d'un ensemble de catastrophes naturelles, la disparition de l'espèce humaine, mais le moment de cet événement reste inconnu et fait l'objet de suppositions. Les signes annonciateurs sont classés en plusieurs catégories temporelles - signes éloignés, intermédiaires ou proches - chacune d'entre elles renvoyant à des évènements plus ou moins précis. Ces textes font mention du Mahdi, une figure religieuse particulièrement importante chez les Chiites, qui dans la fin des temps, luttera contre l'Antéchrist en prenant la tête des armées et vaincra l'ennemi intérieur qui sème la discorde et la guerre civile. Cette figure a été reprise politiquement et religieusement, plusieurs autorités revendiquant le titre de Mahdi tout au long de l'histoire. L'interprétation contemporaine des textes apocalyptiques fait l'objet de la seconde partie de l'article qui insiste sur l'Antéchrist. Les auteurs montrent la prégnance de ces discours dans le cyberespace, l'un des vecteurs essentiel de transmission. Des érudits et des autorités musulmans diffusent leurs analyses et leurs interprétations apocalyptiques, qu'ils tentent de repérer dans la situation contemporaine à partir de positionnements religieux, politiques ou faisant appel à des théories complotistes, reprises ou critiquées sur de nombreux sites, mais convergentes avec d'autres perspectives rapportées en Occident. Cette multiplicité de discours et d'opinions peut en conclusion être classée selon plusieurs catégories (orthodoxe, postorthodoxe spéculatif ou postorthodoxe syncrétiste) qui démontrent la flexibilité et l'hybridité des perspectives apocalyptiques, en remaniement perpétuel, véhiculées grâce aux technologies d'information et de communication contemporaines. 
La notion d'apocalypse peut aussi se distancer complètement de sa matrice religieuse pour devenir un procédé rhétorique visant à caractériser une situation ou un problème dont les répercussions sont potentiellement catastrophiques. Laurence Charton illustre cette perspective dans le domaine sociodémographique avec une analyse des projections et des prédictions sur l'évolution des populations mondiales ou plus localisées, alternant entre la menace d'une surpopulation ou celle d'une sous-population au cours de l'histoire. Depuis les travaux de Malthus, au XVIII siècle, qui reprenait les figures majeures du texte de I'Apocalypse de Jean de Patmos, jusqu'aux discours de l'ex-vice-président américain Al Gore, de nombreux chercheurs, hommes politiques et instances internationales ont ainsi attiré l'attention sur les répercussions sociales, alimentaires, environnementales et politiques que pourrait entraîner l'augmentation incontrôlée de la population mondiale, suggérant des solutions diverses pour atténuer cette éventualité. À l'inverse, plus récemment, les discours catastrophistes se sont orientés vers l'analyse des effets de la sous-population liée à la baisse de la fécondité et au vieillissement de la population, donnant naissance à un courant théorique en démographie défini comme une «démographie apocalyptique» qui fait l'objet de critiques nombreuses, tout comme les prédictions sur la surpopulation et dont la réalisation est toujours reportée dans le temps. Les références à l'apocalypse, sous-jacentes à ces études et à leur diffusion dans les médias, suggèrent que ce type de notion continue à remplir une fonction discursive sensationnaliste, mais qui cache des intérêts économiques et politiques qu'il convient de déchiffrer.

Parmi les évènements contemporains qui ont suscité une effervescence apocalyptique, l'épidémie du VIH/sida occupe une place prépondérante, rejoignant le cortège des fléaux historiques, en particulier la Peste Noire qui avait été interprétée à son époque comme l'un des signes les plus évidents de la fin des temps. L'article de Joseph J. Lévy en dégage les différentes déclinaisons. En premier lieu, on retrouve dans les discours sur le VIH/sida la reprise des thèmes religieux apocalyptiques qui nourrissent les courants fondamentalistes et moralistes aux États-Unis, mais dont l'influence se fait sentir, à travers les missionnaires, dans des régions géographiques isolées ou l'épidémie est peu diffusée ou, au contraire, plus répandue et associée à d'autres contextes problématiques. L'épidémie apparaît alors comme une punition divine suite à la transgression des interdits sexuels bibliques et l'un des signes annoncés dans les textes apocalyptiques. Ces interprétations ne font pas I'unanimité, des théologiens leur opposant une perspective plus conforme au message évangélique de la sollicitude. Des penseurs contemporains, tout en refusant la dimension religieuse, inscrivent, quant à eux, l'épidémie dans la longue liste des apocalypses qui menacent I'humanité contemporaine. Cette notion fait l'objet de critiques qui remettent en question cet usage hyperbolique arrimé à une vision hégémonique et binaire. Au contraire, ils préconisent une approche naturaliste de l'épidémie et le retour à des valeurs éthiques de responsabilité et de justice. À cet égard, les contre-discours provenant des récits autobiographiques, des romans et des témoignages contribueraient à resituer l'épidémie dans un contexte expérientiel, plus à même de révéler les déterminants sociaux 
et économiques. En dernier lieu, les images apocalyptiques servent d'amorce sensationnaliste dans le reportage médiatique sur l'évolution de l'épidémie aux dépends d'une analyse plus objective et plus rationnelle.

La question de la rationalité des idéologies apocalyptiques est ensuite soulevée par Viviane Lew dans son texte sur les enjeux de santé mentale. Ceux-ci sont envisagés de plusieurs points de vue. L'auteure survole en premier lieu les doctrines de sectes apocalyptiques, passées et actuelles, des VieuxCroyants russes à la secte japonaise Aum Shirinkoyo qui se fondent sur des discours faisant appel à des référents bibliques, bouddhistes, politiques ou empruntés aux perspectives New Age, montrant, pour tous ces cas, comment ces systèmes de pensée aboutissent au recours à des stratégies violentes et à des suicides collectifs. Pour comprendre ces comportements, Viviane Lew analyse la source de l'emprise charismatique des leaders de ces sectes sur leurs disciples. Le profil psychologique des leaders montre la présence d'un fort narcissisme associé à une identification à des personnages religieux illustres ou surnaturels qui fondent leur autorité et des traits de personnalité paranoïdes et mégalomaniaques. Les mécanismes de contrôle mis en place pour assurer le maintien du renfermement de la secte vis-à-vis du monde extérieur font appel à des stratégies de surveillance multiples, de la séduction aux punitions, mais les recherches indiquent des résultats contradictoires quant à l'état mental des adhérents à la secte. Les préoccupations de fin de monde qui se retrouvent dans la société contemporaine (risques technologiques, détérioration de l'environnement, épidémies, etc.) ne sont pas non plus exempts de répercussions sur la santé mentale (anxiété, stress, dépression, etc.). Ils peuvent aussi être présents, en particulier chez les jeunes. En dernier lieu, des délires apocalyptiques (religieux ou profanes) se retrouvent aussi chez des patients psychiatriques souffrant de diverses formes de schizophrénie et ce, dans différents pays. Ces patients peuvent s'identifier à des personnages religieux célèbres et tomber dans la violence. Ce tour d'horizon suggère que les idées apocalyptiques jouent un rôle important dans les modulations de la santé mentale, ce qui demande de penser à des interventions pour atténuer leurs répercussions sur la population.

Les trois derniers articles composant notre dossier considèrent la fin du monde dans des perspectives culturelles (littérature, bande dessinée, cinéma). Patrick Bergeron et Antonio Domínguez Leiva s'intéressent à I'une des principales icônes contemporaines de l'apocalypse: le zombie. Ce cadavre animé, issu du folklore haïtien et vaudou, est devenu une figure centrale du cinéma d'horreur après que le réalisateur américain George $A$. Romero en eut fait, dans La nuit des morts-vivants (1968), un dangereux prédateur anthropophage. Le retour en force du zombie dans la culture populaire depuis le début du XXI ${ }^{\text {e }}$ siècle a incité les deux collaborateurs à considérer la place occupée par ce dernier dans les genres narratifs traditionnels et dans le roman graphique. Patrick Bergeron dresse un état des lieux de la présence du zombie dans la littérature contemporaine en se penchant d'abord sur ses origines. Contrairement au vampire, le zombie ne doit pas son succès populaire à la littérature, mais au cinéma, grâce à la trilogie séminale de George A. 
Romero - Night of the Living Dead (1968), Dawn of the Dead (1978) et Day of the Day (1985). Ces films réinterprètent la figure folklorique du zombie haïtien pour en faire une créature apocalyptique dans le prolongement de celles imaginées par Richard Matheson dans I Am Legend (1954), roman qui permit de délimiter plusieurs paramètres de la fiction zombie, notamment sa composante survivaliste (I'intrigue est centrée sur un survivant et ses efforts pour résister au fléau décimateur). Même si les préfigurations littéraires du zombie sont légion (depuis Frankenstein, les revenants sont des figures très présentes au sein de la littérature fantastique), ce dernier relève accessoirement de la littérature. En fait, l'appropriation littéraire du zombie (un phénomène dont l'essor date d'une quinzaine d'années) n'a pas encore donné lieu à un classique de la trempe du chef-d'œuvre de Bram Stoker, Dracula, ni à des œuvres-cultes comparables aux premiers films de Romero et à la série développée par Robert Kirkman, The Walking Dead. Les choses sont peutêtre sur le point de changer, car de Max Brooks à Colson Whitehead en passant par Alden Bell et Julien Péluchon, certains auteurs ont dépassé la stéréotypie gore et baroque pour élaborer une littérature de zombie qui, outre son apocalyptisme, présente d'étonnantes qualités formelles. Dans la deuxième partie de son article, Bergeron propose cinq catégories pour baliser cette production littéraire surtout connue des adeptes du genre.

Antonio Domínguez Leiva se penche pour sa part sur l'apocalypse zombie dans les romans graphiques. Pratiquement absent du $9^{e}$ art au moment où la zombie-manie battait son plein entre la fin des années 1960 et le milieu des années 1980, le mort-vivant romérien a envahi la bande dessinée au point d'en faire l'un des plus importants réceptacles de la renaissance zombie. La bande dessinée de zombies est devenue un sous-genre majeur et très rentable du retour en force des comics d'horreur. Cauchemar graphique ultime, le zombie bédéistique fait la synthèse des grandes anxiétés culturelles du monde contemporain. Après avoir perdu son caractère vaudou pour s'ancrer dans la psychose nucléaire de la Guerre froide, il s'est amalgamé aux motifs et mythèmes de la science-fiction eschatologique (la ville en ruines, I'anomie sociale, le ré-ensauvagement de l'homme, la pandémie, le cannibalisme, le grand-guignolesque gore, la paranoïa vis-à-vis des appareils idéologiques d'État, etc.). Leiva examine les conditions dans lesquelles la figure du zombie est «ressuscitée» au lendemain des attentats du 11 septembre 2001. Plus que tout autre monstre, le zombie contemporain est le signe d'un renversement radical du monde, qui force ce dernier à se transformer à son image. Leiva poursuit sa réflexion en considérant l'indétermination de la pandémie, une topique romérienne dont certains créateurs s'éloignent, puis il commente les principales modalités de la bande dessinée de zombies: I'hybris tragique, le nihilisme, I'humour noir, de même que ses liens avec la contre-culture, les grandes figures marginalisées tels les clochards et junkies, la dystopie et I'iconographie macabre occidentale. En dernière analyse, l'apocalypse zombie dans le roman graphique apparaît comme la représentation d'un Jugement dernier qui aurait lamentablement échoué, car toute rédemption et parousie y sont inconcevables et parce que n'y subsistent que les corps pseudoimmortels des damnés. 
Il est aussi question de réactualisation des imaginaires de la fin dans l'article de Bertrand Gervais, mais à I'intérieur du $7^{\mathrm{e}}$ art. Nous assistons, ces dernières années, à une multiplication des superproductions hollywoodiennes exploitant un canevas apocalyptique. La fin du monde génère d'importantes recettes au box-office. En marge des apocalypses traditionnelles, qui recouvrent un large spectre, on retrouve des apocalypses intimes, qui sont moins spectaculaires sur le plan de la représentation, mais se rattrapent par leur efficacité symbolique. Celle-ci provient de leur entrecroisement du singulier et du collectif, et de leur propos non pas littéral mais allégorique. Les apocalypses intimes promènent un regard chirurgical sur les drames familiaux. Gervais s'attarde à deux illustrations: The Tree of Life (2010) de Terence Malick et Melancholia (2011) de Lars von Trier. Dans le premier cas, le cinéaste fait intervenir un imaginaire de la fin chrétien, inspiré du transcendantalisme américain et mettant en équilibre les tensions entre la vie humaine, dans ses imperfections et ses violences, et le devenir humain, éclairé par la présence du sacré. Dans le second exemple, le cinéaste propose une cinématographie nihiliste, à forte teneur psychanalytique, du moins si on la lit à la lumière de la symptomatologie freudienne de la mélancolie. Plutôt que de jouer la destruction de la planète sur un mode chrétien et sacré comme l'a fait Malick, Trier exploite un imaginaire de la fin d'une grande contemporanéité en ce qu'il fait de l'interruption et de la répétition ses principes dominants.

Nous avons choisi de clore ce dossier par une note de recherche de Valentina Miraglia, qui fait écho à l'article de Bertrand Gervais en étudiant le caractère apocalyptique du cinéma de propagande nazie. Le court-métrage de Kurt Rupli, Das Wort aus Stein (1939), présage selon Miraglia les bombardements qui transformeraient Berlin et les grandes villes d'Allemagne en immenses champs de ruines.

Ainsi, même si elle n'a jamais eu lieu dans les faits, la fin du monde a depuis longtemps généré des discours apocalyptiques qui montrent les efforts de I'humanité pour garder une emprise - réelle? illusoire? - sur un destin qui bien souvent la dépasse et la met en présence de potentialités hostiles, de nature pourtant à lui révéler le sens qu'elle doit donner à ses actions sur terre. Tirant profit de l'intensification de ces discours autour de 2012, ce dossier de Frontières a voulu faire le point sur la question et jeter un éclairage pluridisciplinaire sur une composante résolument marquante du III ${ }^{e}$ millénaire. Ce n'est évidemment qu'un début, car peu de fins présentent un caractère aussi peu définitif que la fin du monde.

\section{NOTES}

1 B. Sergent (2012). La fin du monde. Treize légendes des déluges mésopotamiens au mythe maya, Paris, Librio, coll. «Document», p. 87.

2 S. Pearson (2006). A Brief History of the End of the World. From Revelation to Eco-Disaster, Londres, Robinson, p. 3 ; nous traduisons. 